\title{
Interactive Poster: Illustrating Different Convection Velocities of Turbulent Flow
}

\author{
Timothy Urness* Victoria Interrante \\ Department of Computer Science
}

\author{
Ellen Longmire Ivan Marusic Bharathram Ganapathisubramani \\ Department of Aerospace Engineering and Mechanics
}

University of Minnesota

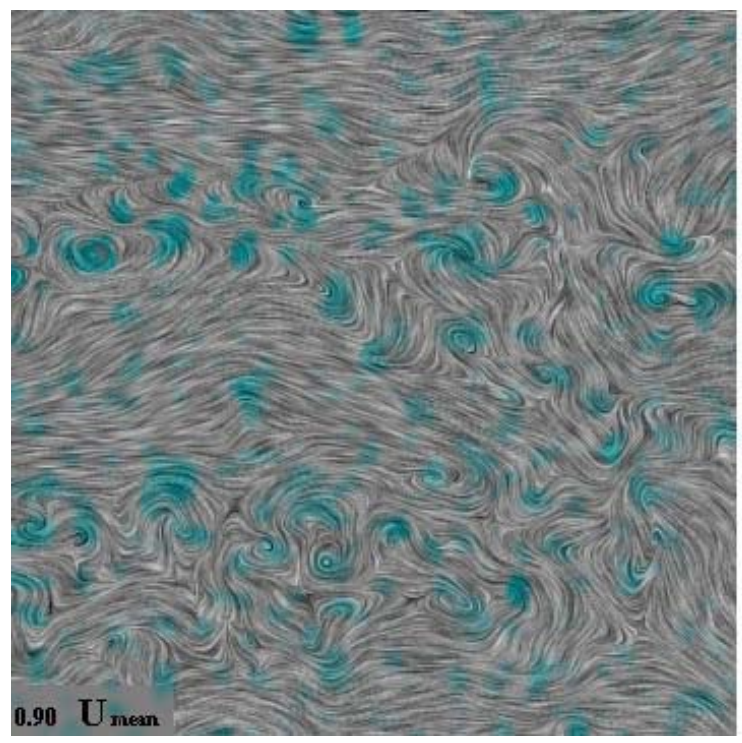

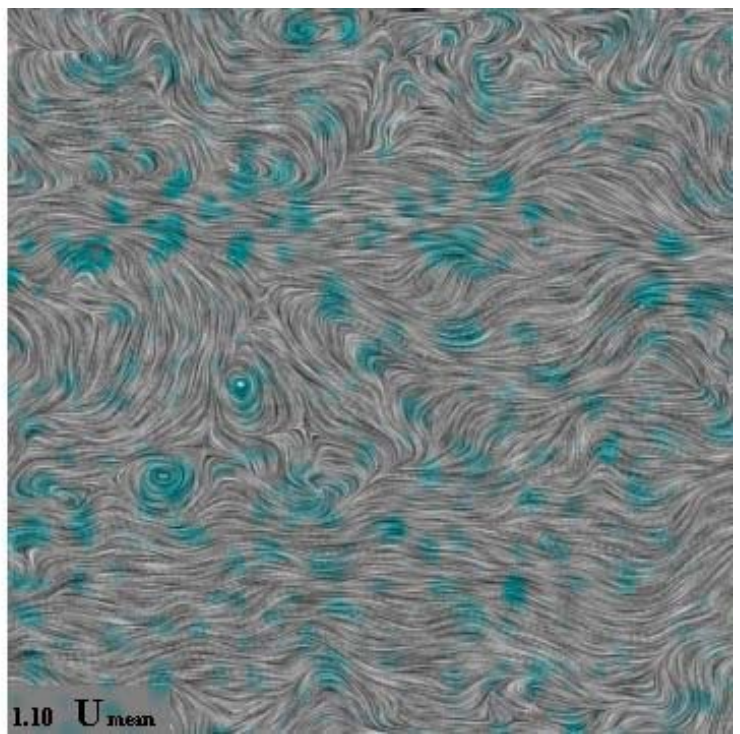

Figure 1: In-plane velocity field with swirl strength superimposed. The value shown in the lower left hand corner is the percentage of the average streamwise velocity that has been subtracted from each vector. Swirling streamlines appear and disappear at different convection velocities.

\section{Abstract}

The visualization of any vector field is dependent on the relative velocity of the observer. In experimentally generated vector fields, the average value of the streamwise component of the global vector field is typically calculated and subtracted from each vector. We demonstrate that the resulting image, critical points, and vector field features are greatly influenced by the magnitude of the value subtracted from the streamwise velocity.

Keywords: flow visualization, texture

\section{INTRODUCTION}

Stereoscopic particle image velocimetry (PIV) is a technique that can be used to experimentally measure instantaneous components of a velocity field in a plane of a turbulent boundary layer in a moderate to high Reynolds number flow. Visualization methods are of critical importance to facilitating the understanding and analysis of results from PIV experiments [4].

\section{Visualization}

Textures, such as line integral convolution (LIC), have traditionally been used to visualize vector fields for the purpose of analyzing the

*e-mail: urness@cs.umn.edu form and behavior of flow and to infer the underlying behavior of experimentally-generated flow fields $[1,3]$. The use of textures allows for a consistent and highly-detailed representation of a vector field allowing an observer to both analyze and better understand the dynamics of fluid flow.

In addition to local velocity magnitude and direction, researchers in turbulent flow have long been interested in a deeper understanding of additional vector and scalar distributions that contribute to theories of drag and the formation of vorticies and vortex packets. Specific scalar fields such as swirl strength, used to analyze the rotation in the form of a coherent vortex, are significant components for the analysis of turbulent flow [2].

Visualizing the PIV vector field as it is originally obtained would prove to be visually uninformative as the image would be dominated by the magnitude of the vectors in the streamwise direction. To account for this phenomenon, the average value of the streamwise component of the global vector field is typically calculated and subtracted from each vector.

\section{Different Convection Velocities}

Subtracting a value of the streamwise component of the global vector field changes the relative velocity of the observer. While this allows for the visualization to be effective, the resulting flow is specific only to that relative velocity. The resulting image, critical points, and vector field features are greatly influenced by the 


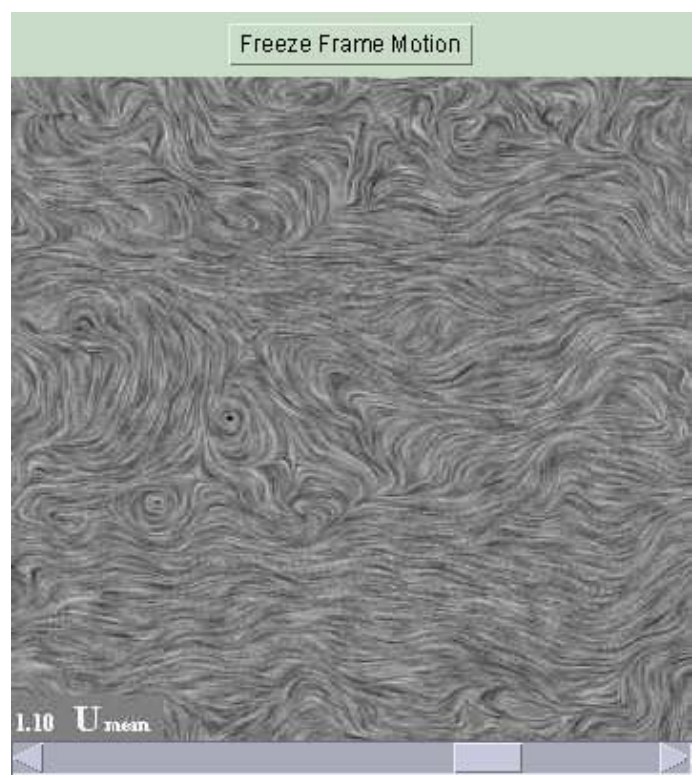

Figure 2: An interactive java applet

magnitude of the value subtracted from the streamwise velocity.

Swirl strength, however, is Galilean invariant and does not vary with the magnitude subtracted from the streamwise velocity. Figure 1 shows two different reference velocities, 90 percent of the streamwise velocity (left) and 110 percent of the streamwise velocity (right). In the leftmost image, the swirling eddies near the bottom left of the flow field are evident only because we have chosen a frame convecting at a speed that matches the convection velocity of those eddies. To illustrate this phenomenon, the swirl strength can be overlaid on a LIC animation computed using a range of relative velocities to determine under what conditions locations with large swirl strength coincide with swirling streamlines.

\section{Applications}

We supply both an mpeg animation and an interactive java applet at http://www.cs.umn.edu/ urness/anim.html

In the mpeg animation, LIC is applied to PIV data and superimposed in color is the swirl strength, which corresponds to vortex cores. The animation runs through a sequence of LIC images in which the magnitude subtracted from the streamwise velocity is gradually incremented. At different moments during the animation, the swirling eddies of the flow field are only apparent because a frame convecting at a speed that matches the convection velocity of those eddies is being displayed.

The java applet (figure 2) allows the user to use the slider at the bottom of the applet to select different magnitudes to be subtracted. The number in the lower left hand corner is the percentage of the average value of the streamwise component that is being subtracted from each vector in the field. The user can also select to "freeze frame motion" to stop the animation and focus only on static LIC images.

\section{ACKNOWLEDGEMENTS}

We would like to acknowledge Kirti Kesavarapu for his help in the development of the software used in this project. This research was supported by a grant from the National Science Foundation (CTS0324898).

\section{REFERENCES}

[1] Brian Cabral and Leith (Casey) Leedom. Imaging vector fields using line integral convolution. In Proceedings of SIGGRAPH 93, pages 263-269. ACM, ACM Press / ACM SIGGRAPH, 1993.

[2] Bharathram Ganapathisubramani, Ellen K. Longmire, and Ivan Marusic. Characteristics of vortex packets in turbulent boundary layers. Journal of Fluid Mechanics, 478:35-46, 2003.

[3] Detlev Stalling and Hans-Christian Hege. Fast and resolutionindependent line integral convolution. In Proceedings of SIGGRAPH 95, pages 249-256. ACM, ACM Press / ACM SIGGRAPH, 1995.

[4] Timothy Urness, Victoria Interrante, Ivan Marusic, Ellen Longmire, and Bharathram Ganapathisubramani. Effectively visualizing multivalued flow data using color and texture. In Proceedings of IEEE Visualization 2003, pages 115-121, 2003. 